\section{Redes de atenção às urgências e emergências: pré-avaliação das Unidades de Pronto Atendimento (UPAs) em uma região metropolitana do Brasil}

\section{Urgent and emergency care networks: a pre-evaluation of the First Aid Units (UPAs) in a metropolitan region of Brazil}

Greciane Soares da Silva 1

Isabella Samico 2

Luciana Santos Dubeux 3

Eronildo Felisberto 4

\begin{abstract}
Objectives: an exploratory evaluative study of the UPAs in a metropolitan region of Brazil in 2011.

Methods: the intervention was described, the logical model outlined, along with the involvement of stakeholders, and evaluation questions drawn up. The three interactive phases approach was carried out using document analysis, interviews and consensus conferencing. The logical model was based on building up a matrix of criteria and indicators that was submitted to a committee of key informants with a view to obtaining consensus.

Results: the matrix of criteria and indicators resulting from the consensus comprises three levels of analysis (health care, inter-institutional interaction and management) with 41 criteria and 74 evaluation indicators. Based on the logical model, the consensus conference, the matrix of criteria and indicators and the systematized considerations of interested parties, fourteen evaluation questions were drawn up.

Conclusions: the UPAs are capable of carrying out evaluations, since it was found that the elements identified in the logical model are conducive to the conditions that the intervention requires in order to meet its aims and objectives.
\end{abstract}

Key words Health evaluation, Health systems, Health management
1-4 Instituto de Medicina Integral Prof. Fernando Figueira. Rua dos Coelhos, 300. Boa Vista. Recife, PE, Brasil. CEP: 50.070-550. E-mail: greciane@imip.org.br.

\section{Resumo}

Objetivos: um estudo avaliativo, exploratório das UPAs na região metropolitana do Recife, no ano de 2011.

Métodos: considerou-se a descrição da intervenção, o delineamento do modelo lógico, o envolvimento dos interessados e a construção de perguntas avaliativas. Realizou-se abordagem de três fases interativas, utilizando, para sua operacionalização, a análise documental, entrevistas e Conferência de Consenso. O modelo lógico elaborado subsidiou a construção de matriz com critérios e indicadores, que foi submetida a um comitê de informantes-chave para obtenção do consenso

Resultados: a matriz de critérios e indicadores resultante do consenso é composta de três niveis de análise (assistência à saúde, integração interistitucional e gestão) com 41 critérios e 74 indicadores avaliativos. Com base no modelo lógico, na Conferência de Consenso, na matriz de critérios/indicadores e nas considerações sistematizadas dos interessados, foram elaboradas 14 perguntas avaliativas.

Conclusões: as UPAs encontram-se adequadas à rea-lização de avaliações, pois se verificou que os elementos identificados no modelo lógico são condizentes com as condições que a intervenção possui para alcançar suas metas e objetivos.

Palavras-chave Avaliação em saúde, Sistemas de saúde, Gestão em Saúde 


\section{Introdução}

A superlotação nas portas das emergências hospitalares é um fenômeno bem conhecido dos gestores, gerentes e usuários. Com seu impacto negativo para os pacientes, essa superlotação constitui um motivo de crítica ao modelo assistencial à saúde, no Brasil causando problemas de difícil gerenciamento. ${ }^{1}$ Para reverter esse quadro nas unidades de emergência, o Ministério da Saúde mediante a Portaria GM n ${ }^{\circ}$ 2.048/2002, publica o Regulamento Técnico dos Sistemas Estaduais de Urgência e Emergência, estimulando a implantação de redes regionalizadas de atendimento, possibilitando uma melhor organização da assistência à saúde. ${ }^{2}$

As médias e grandes regiões metropolitanas têm optado pela organização de uma rede assistencial às urgências através da atenção básica seguindo as Políticas Nacionais de Atenção às Urgências (PNAU) e de Humanização (PNH). ${ }^{1}$ Como expansão da rede de atendimento às urgências e emergências, tem-se as Unidades de Pronto Atendimento (UPAs), integrantes do componente pré-hospitalar fixo das Redes de Atenção Integral às Urgências. Configuram-se como unidades de saúde não hospitalares com complexidade intermediária que visam estabelecer a relação das unidades básicas de saúde e da Estratégia Saúde da Família com a rede hospitalar. No processo de regionalização, a sua integração ao Serviço de Atendimento Móvel de Urgência (SAMU) e à atenção básica diferencia a UPA dos demais serviços de pronto atendimento.1-3

No âmbito de suas atribuições, as UPAs devem atender a demandas durante $24 \mathrm{~h}$, realizar a classificação de risco dos pacientes, resolver os casos de média complexidade, estabilizar os casos graves, como também fornecer retaguarda às unidades de atenção básica. Devem ainda possuir estrutura física, recursos humanos e tecnologia compatíveis com a sua função na assistência à saúde da população. ${ }^{1,4}$

As UPAs desempenham papel importante no atendimento secundário à saúde, contribuindo também para a diminuição da procura pelo atendimento nos hospitais de grande porte. Essas unidades constituem porta de entrada aos serviços de saúde, podendo resolver o caso ou, dependendo da necessidade, encaminhar para internação hospitalar ou redirecionar para a atenção básica.1,2,4 Com a intenção de desafogar as grandes emergências dos hospitais públicos, possuem como características principais o encaminhamento de pacientes em estado grave para hospitais, o funcionamento em horário integral, o atendimento pediátrico, clínica médica e odontológica, 5 além de outras necessidades locorre- gionais, como é o caso das urgências traumáticoortopédicas.

No Estado de Pernambuco, a implantação das UPAs, vem exercendo papel relevante na reestruturação da rede de urgência e emergência, provocando importante debate entre gestores estaduais, municipais e docentes/pesquisadores do campo da saúde pública, que têm considerado o tema como prioritário no debate da agenda política sobre a gestão e a organização do Sistema Único de Saúde (SUS). O Estado, atualmente, conta com quinze UPAs, sob a gestão da Secretaria Estadual de Saúde, sendo treze delas localizadas na região metropolitana do Recife e duas no Agreste, tendo sido o início do funcionamento das mesmas entre os meses de janeiro e setembro de 2010 (11) e janeiro e fevereiro de 2011 (03).

Considerando, portanto, a relevância da inserção desse equipamento de saúde no Sistema Único de Saúde (SUS) estadual, a necessidade de qualificação dos processos e instrumentos de gestão desse novo dispositivo, o tempo de implantação das UPAs, a necessidade de compreensão acerca de suas fortalezas e fragilidades e sua significância na organização do subsistema em que se constitui a rede de urgência e emergência, a prioridade do tema na agenda pública da gestão em saúde no Estado de Pernambuco e, a possibilidade que os estudos de avaliação de políticas, programas e serviços apresentam de ampliar a capacidade de gestores em tomar decisões com maior eficiência, torna-se oportuno realizar estudos avaliativos na perspectiva de contribuir para o aperfeiçoamento da intervenção e a melhoria da qualidade dos serviços ofertados.

O estudo de avaliabilidade (EA) ou pré-avaliação compreende as ações que precedem a etapa inicial do processo avaliativo para determinar se um programa está pronto para receber uma avaliação num dado momento, proporcionando aos interessados um maior entendimento sobre o mesmo. Além de identificar áreas prioritárias para avaliação, pode, através de uma técnica de consenso, verificar a diversidade da compreensão dos componentes da intervenção entre os envolvidos, construindo entendimentos sobre as características, objetivos, expectativas e necessidades de informação sobre uma intervenção. 6-9 O EA foi idealizado na década de 1970 por Joseph Wholey, como um método de análise para programas que se encontravam em estágio insuficiente para se submeterem a avaliações, maximizava a oportunidade, potencialidade, contribuindo para uma maior utilidade dos estudos avaliativos. 4,10 Os produtos de um EA propiciam a descrição do programa e das questões fundamentais 
a serem utilizadas na avaliação, com consenso entre os interessados sobre esses aspectos. $7,11,12$

Portanto, o presente artigo tem como objetivo apresentar os resultados de uma pré-avaliação das Unidades de Pronto Atendimento da região metropolitana do Recife, considerando as seguintes questões: Quais são os componentes fundamentais para o funcionamento dessas Unidades? Quais os critérios e indicadores considerados importantes pelos interessados na intervenção na realização de uma futura avaliação? Quais as áreas da intervenção que precisam ser avaliadas?

\section{Métodos}

Foi realizado um estudo de avaliabilidade em cinco Unidades de Pronto Atendimento da região metropolitana do Recife no período de fevereiro a junho de 2011. Com base em Vanderheyden et al.11 este artigo possui três fases interativas (Figura 1): 1) construção e sistematização do modelo lógico; 2) estabelecimento do consenso com os interessados sobre os elementos-chave do modelo lógico para avaliações futuras; e 3) desenvolvimento de uma matriz de critérios/indicadores e de questões avaliativas.

A coleta de dados foi subsidiada pela análise dos seguintes documentos normativos: Portaria GM/MS $\mathrm{n}^{\mathrm{o}} 1.020 / 2009 ; 4$ Portaria GM no $1.863 / 2003 ; 13$ Portaria GM/MS 2.048/2002;2 Política Nacional de Urgência; 14 Contrato de gestão entre o Estado de Pernambuco e a Fundação Professor Martiniano Fernandes - IMIP Hospitalar; 15 relatórios mensais das UPA;16 contribuições e reflexões dos informantes-chave, integrantes do quadro de profissionais das unidades selecionados em função de seu protagonismo no contexto de implantação da intervenção. Os informantes-chave foram quatro coordenadores, quatro médicos, dois enfermeiros, quatro assistentes sociais e dois especialistas em avaliação; estes últimos foram selecionados pela inserção nas áreas de gestão e avaliação e também pela produção científica sobre o tema em questão. ${ }^{17}$

Neste estudo para descrever o objeto de avaliação assumiu-se a UPA como intervenção, uma vez que é "constituída pelo conjunto dos meios (físicos, humanos, financeiros, simbólicos) organizados em um contexto específico, em um dado momento, para produzir bens ou serviços com o objetivo de modificar uma situação problemática". ${ }^{18}$ Como etapa inicial, foi realizada uma análise documental para o delineamento do modelo lógico, considerando as seguintes questões: Quais são os componentes que melhor representam esta intervenção? Que ativi- dades são necessárias para executar esta intervenção? Quais são os recursos disponíveis e necessários para alcançar os objetivos e resultados desta intervenção? Que resultados podem ser alcançados a curto, médio e longo prazo?

Foram analisadas três dimensões da intervenção: a) assistência à saúde, entendida como eixo principal da intervenção, responsável pelo desenvolvimento de ações diretas com os usuários; b) integração interinstitucional - que visa à relação com outras instituições de atenção à saúde do sistema locorregional; e c) gestão, caracterizada pelas funções gerenciais concernentes à organização e administração da intervenção.

Como revérbero do modelo lógico, foi construída uma matriz com dimensões, subdimensões, critérios e indicadores que pudesse transmitir a operacionalização da intervenção de forma a viabilizar uma maior percepção aos diferentes atores envolvidos. ${ }^{19}$ A realização do desenho dessa matriz com os critérios/indicadores é um passo necessário à implementação da avaliação. 20

De posse da sua primeira versão, iniciou-se a segunda fase do estudo. Para esta fase, a técnica aplicada foi a denominada Conferência de Consenso utilizada por Souza et al. 21 e Reis, 17 com adaptação da terceira etapa, uma técnica mista realizada em três etapas que possibilitam a preservação do anonimato e a discussão aberta entre os participantes.

Na primeira etapa, todos os informantes-chave receberam via e-mail um link específico e individual que os direcionava a uma página na web contendo a matriz com as dimensões, subdimensões, os critérios e indicadores de avaliação propostos. Solicitou-se a cada informante-chave que atribuísse um valor de 0 (zero) a 10 (dez), de acordo com o grau de importância, para se avaliar uma UPA. A nota zero era sem importância ou indicativo de exclusão. As respostas de cada informante-chave eram armazenadas individualmente em uma base de dados para posterior análise. O sistema gerenciador de banco de dados utilizado foi o MySQL versão 4.1.22, e o site foi desenvolvido utilizando PHP, versão 5.0, e o Adobe Flash, versão 8.0.

$\mathrm{Na}$ segunda etapa, foi realizado um encontro presencial com os informantes-chave, possibilitando a discussão sobre as dimensões, subdimensões, os critérios e indicadores de avaliação propostos junto com o consolidado das respostas da etapa anterior, além de reflexões à luz das experiências e dos conhecimentos acumulados por cada um. Ao final dessa discussão, foi formada uma nova matriz com as notas e sugestões dos participantes. 


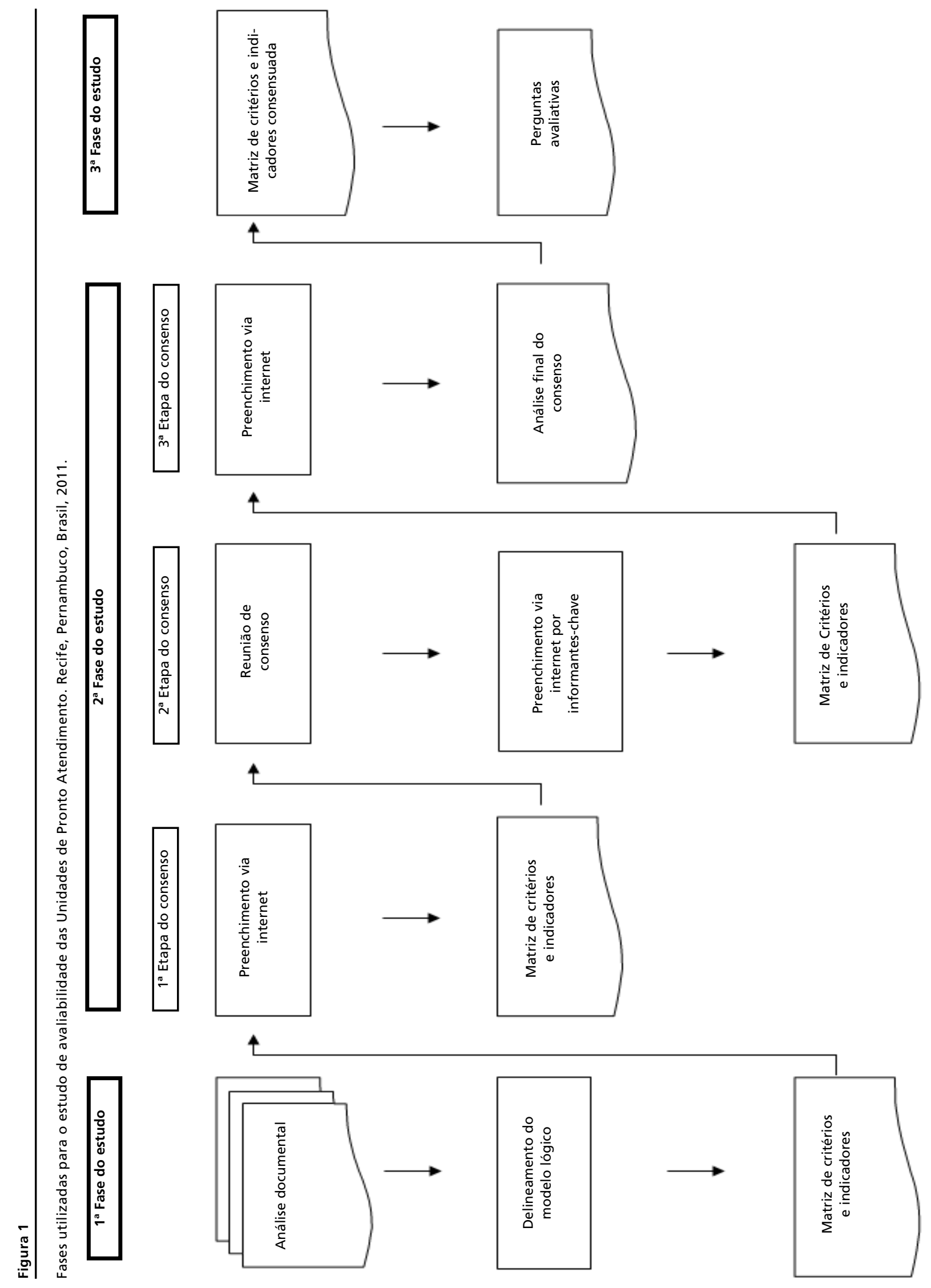


$\mathrm{Na}$ terceira etapa, a matriz e o consolidado das informações discutidas na segunda etapa foram submetidos a todos os informantes-chave com a intenção de se obter o consenso a respeito do produto da etapa anterior, propiciando uma maior aproximação com os conhecimentos, as experiências e sugestões daqueles que não puderam comparecer à segunda etapa.

Após a consolidação das respostas, foram calculados a média aritmética e o desvio-padrão para classificar os componentes da matriz quanto à importância e ao consenso dos informantes-chave, respectivamente. Os pontos de corte usados na classificação foram de sete para a média e de três para o desvio-padrão, com base nos pontos de corte utilizados por Souza et al. 21 e Reis. 17 Desta forma, as dimensões, as subdimensões, os critérios e os indicadores foram enquadrados em quatro grupos da seguinte forma: a) Consensualmente importantes: dimensões, subdimensões, critérios e indicadores com desvio-padrão inferior a três, indicando grau de consenso elevado e média igual ou superior a sete, indicando grau de importância elevado; b) Consensualmente pouco importantes: dimensões, subdimensões, critérios e indicadores com desvio-padrão inferior a três, e média inferior a sete indicando baixo grau de importância; c) Importantes com dissenso: dimensões, subdimensões, critérios e indicadores com média superior ou igual a sete e desvio-padrão maior ou igual a três, indicando que prevaleceu a discordância entre os informantes-chave; d) Pouco importantes com dissenso: dimensões, subdimensões, critérios e indicadores com média inferior a sete e desvio-padrão superior ou igual a três.

A formulação das perguntas avaliativas pelos envolvidos no estudo de avaliabilidade teve como base o enriquecimento adquirido durante o processo de Conferência de Consenso, envolvendo os interessados a partir das seguintes questões: a) cite algumas das suas principais preocupações em relação à UPA, b) em sua opinião, quais são as principais perguntas que você gostaria que uma avaliação respondesse? c) como você usaria as informações produzidas por essas perguntas? (Você utilizaria as respostas para tomar decisões, para lhe trazer esclarecimentos?).22 De posse das respostas, as perguntas avaliativas foram categorizadas segundo o tipo de análise. Essas perguntas não só ajudaram a explicar a lógica da intervenção, como também puderam propiciar uma base para futuras avaliações. 12

O estudo foi aprovado pelo Comitê de Ética em Pesquisa em Seres Humanos do Instituto de Medicina Prof. Fernando Figueira (IMIP), estando de acordo com a resolução 196/96 do Conselho
Nacional de Saúde, registro n² 2160-11.

\section{Resultados}

A análise realizada para construção do modelo lógico (Figura 2) possibilitou visualizar a intervenção de cada dimensão com suas respectivas subdimensões: assistência à saúde (subdimensões: atendimento de urgência e procedimentos diagnósticos), integração interinstitucional (subdimensões: atuação complementar à atenção básica às urgências, SAMU192, unidades de apoio diagnóstico-terapêutico e unidades hospitalares) e Gestão (subdimensões: gestão administrativa, gestão financeira e gestão de qualidade). Para cada dimensão, consideraram-se os insumos, as atividades e os resultados esperados, de tal forma que os elementos tidos como essenciais para representar a operacionalização das Unidades de Pronto Atendimento ficaram traçados em uma lógica que auxiliou os envolvidos numa melhor compreensão dos critérios e indicadores correlacionados a cada dimensão.

A matriz de critérios e indicadores, apresentada na Tabela 1, foi submetida à Conferência de Consenso para sua validação. Na primeira etapa da conferência, houve prevalência do consenso, embora tenha sido destaque o considerável número de dissenso: 47 (28\%) critérios e indicadores. A matriz de critérios e indicadores encaminhada na primeira etapa aos informantes-chave era formada por três dimensões, nove subdimensões, 84 critérios e 84 indicadores. Como resultado desta etapa, pode-se constatar que houve consenso nas dimensões e subdimensões. Dos 84 critérios, 70 foram classificados como importantes consensualmente, um foi classificado consensualmente como não importante e 13 tiveram dissenso na sua classificação; destes, nove foram classificados como não importantes. Quanto aos indicadores, houve dissenso em 34 e, dos 50 indicadores em consenso, 41 foram classificados como importantes.

$\mathrm{Na}$ segunda etapa da reunião presencial compareceram cinco informantes-chave; destes, três haviam participado do primeiro momento. $\mathrm{O}$ encontro propiciou uma melhor percepção sobre o delineamento do modelo lógico da intervenção UPA. Os componentes, as atividades, a estrutura e os resultados do modelo lógico foram identificados nos componentes da matriz de critérios e indicadores submetida ao consenso. As principais contribuições dos informantes-chave foram quanto ao objetivo e ao resultado final da intervenção: o objetivo geral da UPA estaria centrado na ampliação de acesso e na qualidade dos atendimentos de urgência e 


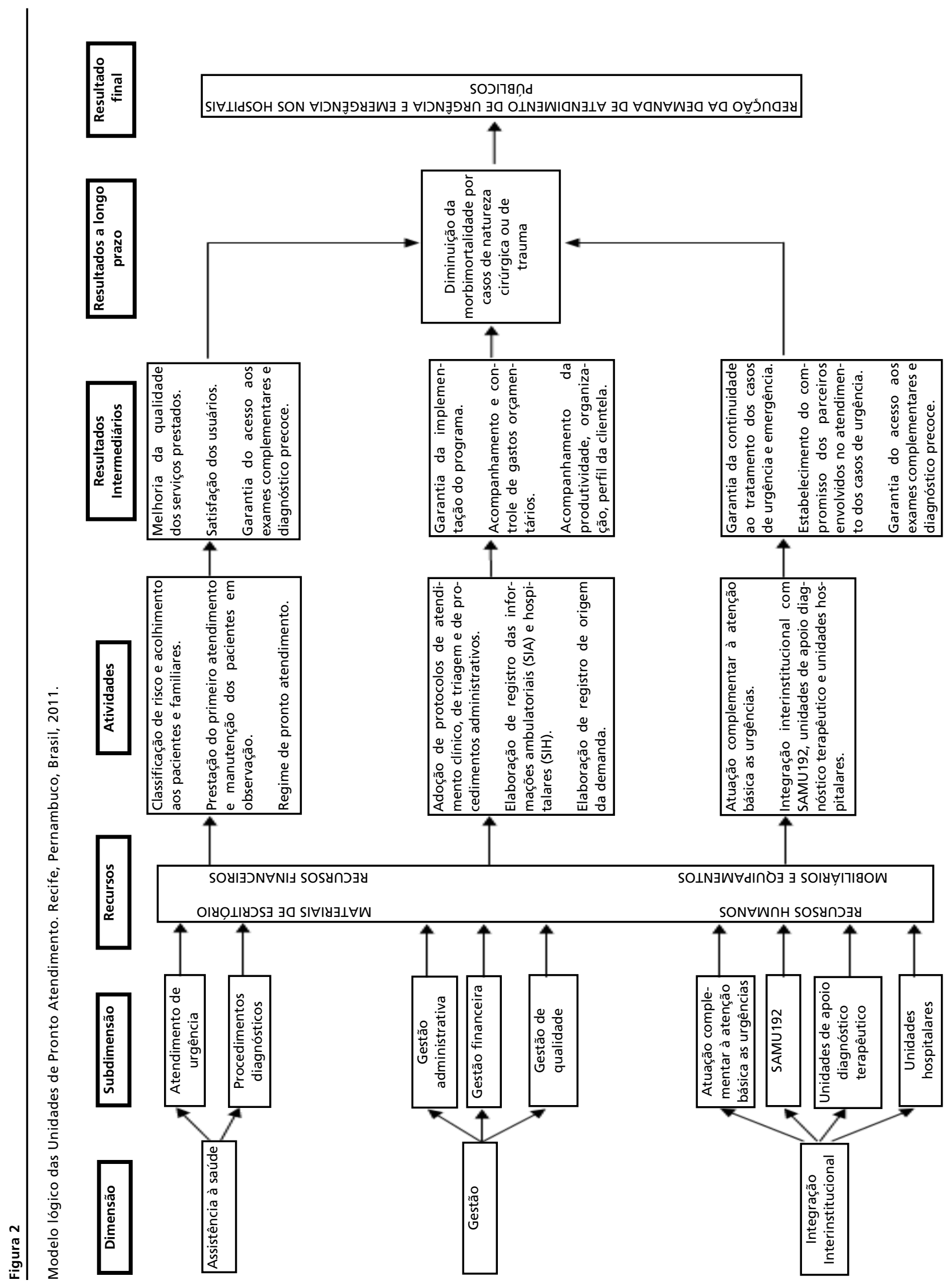


Matriz com critérios e indicadores das Unidades de Pronto Atendimento: resultados do consenso. Recife, Pernambuco, Brasil, 2011.

\begin{tabular}{|c|c|c|c|}
\hline $\begin{array}{l}\text { Dimensão } \\
\text { Assistência à Saúde } \\
\text { Subdimensão } \\
\quad \text { Atendimento de Urgência }\end{array}$ & & & $\begin{array}{l}\bar{x} \pm \mathrm{DP} \\
9,4 \pm 1,2 \\
9,2 \pm 1,2\end{array}$ \\
\hline \multirow[t]{5}{*}{ Critérios } & Indicadores & Indicadores & Critérios \\
\hline & & $\bar{X} \pm \mathrm{DP}$ & $\bar{X} \pm \mathrm{DP}$ \\
\hline & Adequação da sala de recepção/espera & $8,4 \pm 1,6$ & \\
\hline & Adequação dos sanitários do Setor de Pronto Atendimento & $8,2 \pm 1,7$ & \\
\hline & Adequação da sala de classificação de risco & $9,1 \pm 1,4$ & \\
\hline \multirow[t]{6}{*}{ Setor de Pronto Atendimento } & Adequação da sala de atendimento social & $8,9 \pm 1,2$ & $8,1 \pm 2,0$ \\
\hline & Adequação da sala para exame indiferenciado & $7,8 \pm 2,9$ & \\
\hline & Adequação da sala para exame diferenciado - odontologia & $5,6 \pm 4,3$ & \\
\hline & $\begin{array}{l}\text { Adequação do depósito para material de limpeza do Setor de } \\
\text { Pronto Atendimento }\end{array}$ & $7,5 \pm 1,9$ & \\
\hline & Adequação da área externa para desembarque de ambulância & $8,4 \pm 1,8$ & \\
\hline & Adequação da sala de higienização & $7,5 \pm 3,0$ & \\
\hline \multirow[t]{3}{*}{ Setor de Atendimento de Urgência } & Adequação da sala de urgência & $9,2 \pm 1,5$ & $8,2 \pm 1,8$ \\
\hline & Adequação da área para guarda de macas/ cadeira de rodas & $7,2 \pm 2,0$ & \\
\hline & $\begin{array}{l}\text { Adequação do depósito de material de limpeza do Setor de } \\
\text { Atendimento de Urgência }\end{array}$ & $7,2 \pm 1,8$ & \\
\hline & Adequação do posto de enfermagem/ serviços & $8,8 \pm 1,5$ & \\
\hline \multirow[t]{2}{*}{ Setor de Observação } & $\begin{array}{l}\text { Adequação da sala coletiva para leitos de observação com os } \\
\text { respectivos banheiros para pacientes internos }\end{array}$ & $\begin{array}{l}8,7 \pm 1,5 \\
7,2 \pm 3,4\end{array}$ & $8,9 \pm 1,8$ \\
\hline & Adequação dos quartos individuais e respectivos banheiros & & \\
\hline $\begin{array}{l}\text { Estruturação do serviço de acolhimento a } \\
\text { pacientes e familiares }\end{array}$ & Estrutura adequada do serviço de acolhimento & $8,5 \pm 2,5$ & $8,9 \pm 1,1$ \\
\hline Leitos de observação suficientes & Número de leitos de observação & $8,9 \pm 1,3$ & $9,2 \pm 1,2$ \\
\hline Médicos plantonistas & Número de médicos por plantão & $7,9 \pm 2,9$ & $8,7 \pm 1,9$ \\
\hline Enfermeiros plantonistas & Número de enfermeiros por plantão & $8,2 \pm 2,2$ & $9,1 \pm 1,6$ \\
\hline Assistentes sociais plantonistas & Número de assistentes sociais por plantão & $8,8 \pm 1,5$ & $8,9 \pm 1,6$ \\
\hline $\begin{array}{l}\text { Atendimento de casos de natureza cirúrgica ou } \\
\text { de trauma }\end{array}$ & Porcentagem de consultas/mês especificado em contrato & $7,6 \pm 2,6$ & $9,2 \pm 3,3$ \\
\hline Realização de consultas de clínica médica & Porcentagem do número de consultas/mês especificado em contrato & $7,4 \pm 2,6$ & $9,2 \pm 1,5$ \\
\hline Realização de consultas de clínica ortopédica & Porcentagem do número de consultas/mês especificado em contrato & $6,4 \pm 3,9$ & $8,6 \pm 1,8$ \\
\hline Realização de consultas de clínica pediátrica & Porcentagem do número de consultas/mês especificado em contrato & $7,5 \pm 2,6$ & $9,5 \pm 1,6$ \\
\hline $\begin{array}{l}\text { Atendimentos e procedimentos de } \\
\text { enfermagem }\end{array}$ & Porcentagem do número de consultas/mês especificado em contrato & $7,5 \pm 2,6$ & $9,3 \pm 1,6$ \\
\hline Estabilização clínica & Realização de estabilização clínica & $9,1 \pm 1,6$ & $9,4 \pm 2,3$ \\
\hline
\end{tabular}


Matriz com critérios e indicadores das Unidades de Pronto Atendimento: resultados do consenso. Recife, Pernambuco, Brasil, 2011.

\begin{tabular}{|c|c|c|c|}
\hline \multirow[t]{2}{*}{ Critérios } & \multirow[t]{2}{*}{ Indicadores } & \multirow{2}{*}{$\frac{\text { Indicadores }}{\overline{\mathrm{X}} \pm \mathrm{DP}}$} & \multirow{2}{*}{$\begin{array}{c}\text { Critérios } \\
\overline{\mathbf{X}} \pm \mathrm{DP} \\
\end{array}$} \\
\hline & & & \\
\hline \multicolumn{4}{|l|}{ Subdimensão } \\
\hline \multicolumn{2}{|l|}{ Procedimentos diagnósticos } & \multicolumn{2}{|c|}{$9,2 \pm 1,2$} \\
\hline \multirow{7}{*}{ Setor de Apoio Diagnóstico e Terapêutico } & Adequação da sala de eletrocardiografia & $7,2 \pm 2,4$ & $8,7 \pm 1,6$ \\
\hline & Adequação da sala de sutura/curativos & $8,5 \pm 1,6$ & \\
\hline & Adequação da sala de gesso/ imobilização de fraturas & $8,0 \pm 2,8$ & \\
\hline & Adequação da sala de inalação coletiva & $8,2 \pm 2,0$ & \\
\hline & Adequação da sala de aplicação de medicamentos/reidratação & $8,5 \pm 2,0$ & \\
\hline & Adequação das salas de exames de radiologia geral & $8,6 \pm 1,9$ & \\
\hline & Adequação do laboratório de processamento (câmara escura) & $8,2 \pm 2,3$ & \\
\hline \multirow{3}{*}{ Realização de Raios-X } & Adequação do arquivo de chapas & $7,1 \pm 3,3$ & \\
\hline & $\begin{array}{l}\text { Adequação do box de vestiário para paciente e sala de coleta de } \\
\text { material }\end{array}$ & $6,9 \pm 2,2$ & $7,9 \pm 2,1$ \\
\hline & $\begin{array}{l}\text { Porcentagem do número de consultas/mês especificado em } \\
\text { contrato }\end{array}$ & $7,6 \pm 2,9$ & \\
\hline Realização de exames laboratoriais & $\begin{array}{l}\text { Porcentagem do número de consultas/mês especificado em } \\
\text { contrato }\end{array}$ & $7,4 \pm 2,9$ & $8,9 \pm 1,4$ \\
\hline Realização de eletrocardiograma & $\begin{array}{l}\text { Porcentagem do número de consultas/mês especificado em } \\
\text { contrato }\end{array}$ & $7,5 \pm 2,9$ & $9,1 \pm 1,2$ \\
\hline Classificação de risco no serviço & Percentual dos casos classificados quanto ao risco & $7,5 \pm 2,3$ & $8,4 \pm 2,1$ \\
\hline \multicolumn{2}{|c|}{$\begin{array}{l}\text { Dimensão } \\
\text { Integração interinstitucional } \\
\text { Subdimensão } \\
\quad \text { Atuação complementar à atenção básica as urgências }\end{array}$} & \multicolumn{2}{|r|}{$\begin{array}{l}\overline{\mathbf{X}} \pm \mathrm{DP} \\
9,4 \pm 1,2 \\
9,2 \pm 1,2\end{array}$} \\
\hline $\begin{array}{l}\text { Prestação de atendimento médico aos casos } \\
\text { referenciados da Atenção Básica }\end{array}$ & Realização do atendimento & $8,4 \pm 1,4$ & $8,5 \pm 1,3$ \\
\hline Prestação de atendimento médico à noite & Realização do atendimento & $8,5 \pm 1,7$ & $8,5 \pm 1,7$ \\
\hline $\begin{array}{l}\text { Prestação de atendimento médico nos } \\
\text { finais de semana }\end{array}$ & Realização do atendimento & $8,3 \pm 2,9$ & $8,5 \pm 1,7$ \\
\hline $\begin{array}{l}\text { Prestação de atendimento de enfermagem } \\
\text { aos casos referenciados da Atenção Básica }\end{array}$ & Realização do atendimento & $7,9 \pm 2,4$ & $8,5 \pm 1,7$ \\
\hline $\begin{array}{l}\text { Prestação de atendimento de enfermagem } \\
\text { à noite }\end{array}$ & Realização do atendimento & $8,9 \pm 1,4$ & $8,5 \pm 1,7$ \\
\hline $\begin{array}{l}\text { Prestação de atendimento de enfermagem } \\
\text { nos finais de semana }\end{array}$ & Realização do atendimento & $8,5 \pm 2,8$ & $8,5 \pm 1,7$ \\
\hline
\end{tabular}


Matriz com critérios e indicadores das Unidades de Pronto Atendimento: resultados do consenso. Recife, Pernambuco, Brasil, 2011.

\begin{tabular}{|c|c|c|c|}
\hline \multirow[t]{2}{*}{ Critérios } & \multirow[t]{2}{*}{ Indicadores } & \multirow{2}{*}{$\frac{\text { Indicadores }}{\bar{X} \pm \mathrm{DP}}$} & \multirow{2}{*}{$\begin{array}{c}\text { Critérios } \\
\bar{X} \pm \mathrm{DP}\end{array}$} \\
\hline & & & \\
\hline \multicolumn{4}{|l|}{ Subdimensão } \\
\hline SAMU192 & & \multicolumn{2}{|c|}{$8,5 \pm 1,9$} \\
\hline $\begin{array}{l}\text { Realização de atendimento médico aos } \\
\text { pacientes atendidos pelo SAMU } 192\end{array}$ & Realização do atendimento & $8,9 \pm 1,1$ & $9,1 \pm 1,0$ \\
\hline $\begin{array}{l}\text { Solicitação de atendimento médico sempre } \\
\text { que a gravidade/ complexidade dos casos } \\
\text { ultrapassarem a capacidade instalada da } \\
\text { Unidade }\end{array}$ & Realização do atendimento & $7,7 \pm 1,8$ & $7,9 \pm 1,9$ \\
\hline $\begin{array}{l}\text { Realização de atendimento de enfermagem } \\
\text { aos pacientes atendidos pelo SAMU } 192\end{array}$ & Realização do atendimento & $8,9 \pm 1,1$ & $9,0 \pm 1,2$ \\
\hline $\begin{array}{l}\text { Solicitação de atendimento de enfermagem } \\
\text { sempre que a gravidade/ complexidade dos } \\
\text { casos ultrapassarem a capacidade instalada } \\
\text { da Unidade }\end{array}$ & Realização do atendimento & $8,0 \pm 1,6$ & $8,2 \pm 1,7$ \\
\hline
\end{tabular}

Subdimensão

Unidades de apoio diagnóstico terapêutico

Encaminhamentos dos casos não resolvidos

Prestação de atendimento médico à noite

\begin{tabular}{l} 
Dimensão \\
Gestão \\
Subdimensão \\
Gestão Administrativa \\
\hline
\end{tabular}

Setor de Apoio Administrativo

Setor de Apoio Técnico e Logístico
Adequação da sala de direção

Adequação da sala de reuniões

Adequação do arquivo médico

Adequação da sala administrativa/ informática/ ponto/protocolo

Adequação do posto policial

Adequação da área de distribuição (farmácia)

Adequação da área para armazenamento de materiais e equipamentos

Adequação da sala de lavagem e descontaminação dos materiais $\quad 7,6 \pm 3,3$

Adequação da sala de armazenamento e distribuição de materiais $\quad 8,5 \pm 1,3$ esterilizados

Adequação da copa de distribuição

$7,5 \pm 2,4$

Adequação do refeitório de funcionários

$7,7 \pm 2,9$

$7,7 \pm 2,4$

Adequação do almoxarifado

$7,8 \pm 2,3$

$7,7 \pm 1,3$

$5,8 \pm 3,5$

Adequação da sala de utilidades

$7,8 \pm 2,3$

Adequação da sala para equipamentos de geração de energia elétrica alternativa

Adequação da área para gases (cilindros)

$8,4 \pm 1,6$

Adequação da sala para guarda temporária de cadáveres
$9,2 \pm 1,0$

$8,9 \pm 1,3$ 
Matriz com critérios e indicadores das Unidades de Pronto Atendimento: resultados do consenso. Recife, Pernambuco, Brasil, 2011.

\begin{tabular}{|c|c|c|c|}
\hline \multirow[t]{2}{*}{ Critérios } & \multirow[t]{2}{*}{ Indicadores } & \multirow{2}{*}{$\frac{\text { Indicadores }}{\bar{X} \pm \mathrm{DP}}$} & \multirow{2}{*}{$\frac{\text { Critérios }}{\overline{\mathrm{X}} \pm \mathrm{DP}}$} \\
\hline & & & \\
\hline $\begin{array}{l}\text { Dimensão } \\
\text { Gestão } \\
\text { Subdimensão } \\
\text { Gestão Administrativa }\end{array}$ & & \multicolumn{2}{|c|}{$9,2 \pm 1,0$} \\
\hline & Adequação da área externa para embarque de carro funerário & $7,9 \pm 2,4$ & \\
\hline & Adequação do quarto de plantão para funcionários & $8,1 \pm 1,8$ & \\
\hline & Adequação da sala de estar para funcionários & $6,9 \pm 3,3$ & \\
\hline & Adequação dos banheiros para funcionários & $8,0 \pm 1,6$ & \\
\hline & Adequação do vestiário para funcionários & $8,1 \pm 1,8$ & \\
\hline & Adequação dos sanitários para funcionários e acompanhantes & $7,8 \pm 1,9$ & \\
\hline & Adequação da sala para armazenamento temporário de resíduos & $7,4 \pm 2,4$ & \\
\hline & Adequação do abrigo externo de resíduos & $7,5 \pm 2,3$ & \\
\hline Protocolos de atendimento clínico & Adoção de protocolos de atendimento clínico & $8,7 \pm 2,1$ & $9,2 \pm 1,0$ \\
\hline Protocolos de triagem & Adoção de protocolos de triagem & $8,6 \pm 2,3$ & $8,9 \pm 1,7$ \\
\hline $\begin{array}{l}\text { Protocolos de procedimentos administra- } \\
\text { tivos }\end{array}$ & Adoção de protocolos de procedimentos administrativos & $8,5 \pm 1,6$ & $8,6 \pm 1,3$ \\
\hline \multicolumn{4}{|l|}{ Subdimensão } \\
\hline Recursos financeiros & Aplicação de recursos financeiros & $9,0 \pm 1,3$ & $9,0 \pm 1,3$ \\
\hline \multicolumn{2}{|l|}{$\begin{array}{l}\text { Subdimensão } \\
\quad \text { Gestão de Qualidade }\end{array}$} & \multicolumn{2}{|c|}{$9,0 \pm 1,3$} \\
\hline \multirow{2}{*}{$\begin{array}{l}\text { Realização de pesquisa da satisfação do } \\
\text { usuário }\end{array}$} & Envio da pesquisa de satisfação do usuário & $9,2 \pm 1,0$ & $8,9 \pm 1,2$ \\
\hline & Percentual de resolução de queixas & $8,8 \pm 2,7$ & $8,7 \pm 2,7$ \\
\hline \multicolumn{4}{|l|}{ Resolução de queixas } \\
\hline & Percentual de funcionários capacitados no Programa de Educação & $8,8 \pm 1,2$ & $9,3 \pm 1,0$ \\
\hline Profissionais capacitado & Permanente & & \\
\hline
\end{tabular}


emergência, e, como resultado final, tem-se a redução da demanda desses atendimentos nos hospitais públicos.

$\mathrm{Na}$ terceira e última etapa da Conferência de Consenso, a matriz apresentou consenso para três dimensões, nove subdimensões, 77 critérios e 74 indicadores. No tocante às dimensões, a que obteve maior grau de importância foi a assistência à saúde (média $=9,4$ e desvio-padrão $=1,2$ ), seguida pela dimensão gestão (média $=9,2$ e desvio-padrão $=1,0$ ) e pela integração interinstitucional (média $=9,0 \mathrm{e}$ desvio-padrão $=1,5)$. Quanto às subdimensões, a soma das maiores médias se concentrou na mesma ordem das dimensões: dimensão assistência à saúde, seguida da dimensão gestão e da integração interinstitucional.

Quanto aos critérios, as maiores médias se concentraram na dimensão assistência à saúde. Ao final dessa etapa, os critérios relativos à estrutura foram agrupados em setores segundo a natureza funcional. Assim a matriz passa a apresentar 41 critérios para melhor representação.

Dos nove indicadores em dissenso, cinco estavam em assistência à saúde: adequação da sala para exame diferenciado - odontologia; adequação da sala de higienização; porcentagem de consultas de clínicas ortopédica/mês especificada em contrato; adequação do arquivo de chapas; adequação dos quartos individuais e respectivos banheiros. Os outros quatro, que estavam na dimensão gestão, foram: adequação da sala de reuniões; adequação da sala de lavagem e descontaminação dos materiais; adequação da sala de utilidades; adequação da sala de estar para funcionários.

Dos 13 participantes que finalizaram a terceira etapa do consenso, cinco responderam às questões para a elaboração das perguntas avaliativas de forma abrangente, expondo seu ponto de vista sem a preocupação de filtrar prioridades. Com base no modelo lógico, na Conferência de Consenso, na matriz de critérios/indicadores e nas considerações sistematizadas dos interessados, foram elaboradas as seguintes perguntas avaliativas: a) as atividades em sua plenitude são suficientes para o alcance dos resultados? (análise estratégica/produtividade); b) em que medida os recursos são empregados adequadamente para atingir os resultados esperados? (análise de estrutura); c) em que medida os serviços estão adequados para atingir os resultados esperados? (análise de processo); d) quais os fatores relacionados ao contexto organizacional que podem influenciar a implantação da UPA? (análise de implantação); e) em que medida o quantitativo de profissionais está sendo adequado para atender ao resultado esperado da UPA? (análise de estrutura/processo); f) que tipo de conflito ocorre entre os profissionais? Qual a natureza deste conflito? Esses conflitos interferem na qualidade dos serviços? (avaliação de contexto/implantação); g) que impacto as ações de educação em saúde têm na assistência à saúde oferecida pela UPA? (análise dos efeitos/avaliação de impacto); h) é possível produzir serviços de melhor qualidade com os mesmos recursos? (análise de produtividade); i) a UPA está alcançando a população programada? Quais os fatores do contexto locorregional institucional e organizacional que podem influenciar no perfil da demanda dos usuários atendidos na UPA? (análise de implantação); j) em que medida a UPA consegue dar continuidade aos tratamentos de urgência e emergências? (análise de efeitos/monitoramento); 1) qual relação custo-efetividade da intervenção? (análise de rendimentos).

\section{Discussão}

Este estudo mostrou-se importante não só pela contribuição da avaliabilidade para um melhor entendimento da intervenção e da sua apreciação lógica, como também pela capacidade de incluir diferentes atores nos componentes analisados consensualmente. Essa integração promove aprendizado sobre a intervenção e fornece subsídios para valorização e ação a partir dos resultados e conclusões da avaliação. 23 Um dos principais objetivos do EA foi alcançado com a identificação e o envolvimento das pessoas que têm um papel central na intervenção para atender as necessidades reais e que podem fazer uso deste estudo em avaliações futuras. 8,24

O modelo lógico reflete um referencial que permite aos interessados visualizar com mais clareza a operacionalização da intervenção, aumentando a construção de acordos em relação aos componentes do programa e aos resultados da avaliação. $20,25 \mathrm{~A}$ elaboração do modelo lógico permitiu, de forma esquemática detalhar os componentes da intervenção, facilitando a percepção objetiva do funcionamento da mesma para o comitê de avaliadores e profissionais participantes. ${ }^{20,26}$ Além de favorecer o entendimento comum acerca do problema que se quer resolver, possibilitou também a discussão sobre os objetivos, os resultados esperados e o processo necessário para alcançá-los. ${ }^{27}$

Os documentos utilizados para o delineamento do modelo lógico foram os que possibilitaram a construção dos componentes que o constituíram, com variação para algumas metas contratuais, uma 
vez que certas Unidades de Pronto Atendimento possuem metas diferentes diante da realidade locorregional. Por outro lado, pode-se considerar que documentos de programa em fase de criação geralmente são poucos; porém, dependendo de sua complexidade, diferentes tipos de documentos podem surgir.7,12

Após analisar os componentes integrantes do modelo lógico, percebe-se que essa ferramenta tem grande relevância durante todo o EA. Destacam-se os seguintes benefícios: proporciona um melhor entendimento da intervenção; ajuda a divulgar a intervenção de maneira sucinta e objetiva; clarifica responsabilidades para os interessados.

Em relação à análise da lógica da intervenção, observou-se a necessidade da inclusão de mais critérios e indicadores relacionados aos aspectos de processo. A escassez de critérios e indicadores de processo permite que tanto o modelo lógico quanto a matriz de critérios e indicadores sejam passíveis de modificações em qualquer tempo, principalmente pelo fato de os informantes-chave considerarem a dimensão Assistência à Saúde, constituída de muitos processos, sendo também o componente com maior grau de importância para se avaliar uma UPA. Como referido por Vuori:28 “(...) se se quer melhorar a assistência, isso tem que acontecer no nível do processo ou da estrutura. Resultados são, sempre, consequência de alguma coisa. Se os resultados são precários, há que se voltar ao processo e descobrir coisas a serem corrigidas".

Neste estudo, os resultados sugerem que há coerência na relação causal entre os elementos do modelo lógico, ou seja, há plausibilidade da intervenção. Este é um aspecto considerado essencial para um estudo de avaliabilidade ou pré-avaliação, pois, sem essa análise, tanto o modelo lógico como o desenvolvimento das atividades perdem o significado. 29

A apreciação dos componentes realizada através da análise dos informantes-chave envolvidos na Conferência de Consenso levou em consideração as melhores ideias ou decisões a serem tomadas sobre a situação apresentada. ${ }^{30}$ Chama à atenção o fato de que a maior média $(9,4)$ foi para a subdimensão gestão da qualidade e a segunda maior média $(9,2)$ para a subdimensão integração interinstitucional à atenção básica.

Outro achado bastante expressivo diz respeito ao alto grau de dissenso sobre os componentes da matriz de critérios e indicadores na primeira etapa da construção do consenso. A maior parte da reunião presencial com os informantes-chave foi dedicada à discussão sobre o número de dissenso atribuído à essa primeira etapa. $\mathrm{Na}$ ótica dos mesmos, esse dissenso poderia ser reflexo da experiência e interpretação pessoal que cada participante possuía a respeito de critério/indicador, manifestando, assim, a vontade individual de realizar uma própria avaliação da UPA em que atuavam. Além disso, deveria ser considerada a dificuldade de se atribuir uma nota a um critério/indicador que não se tenha em prática no serviço, podendo ter sido esta a causa de algumas notas inferiores a sete, uma vez que muitos profissionais precisam de um parâmetro para realizar a pontuação e esta pode ter sido dada pelo grau de experiência que cada um possui com relação à UPA. Stufflebeam ${ }^{23}$ considera que estabelecer valores não é uma tarefa fácil. É um constante desafio para o trabalho avaliativo. Diferentes interessados em uma organização e membros da sociedade como um todo podem conservar valores conflitantes sobre muitas questões.

O levantamento das suposições apontadas pelos informantes-chave juntamente com a revisão dos pressupostos que fundamentam a intervenção facilitam a identificação das possíveis lacunas da lógica do programa.24,31,32 De acordo com Renger e Titcomb, 32 o termo "lógica subjacente" se refere a uma representação esquemática das causas do problema a ser alvo. Alguns modelos lógicos não conseguem retratar esquematicamente a lógica subjacente da intervenção, tornando difícil compreender a relação entre esta e os elementos da avaliação.

Diante da discussão dos fatores causais relacionados à intervenção, o grupo entendeu que, enquanto intervenção do Ministério da Saúde, a UPA pretende a "redução da demanda de atendimento de urgência e emergência nos hospitais públicos", o que não desconsidera a "diminuição da morbimortalidade por casos de natureza cirúrgica ou de trauma" como resultado a longo prazo, considerada no modelo lógico.

Outro aspecto considerado no debate foi quanto à clareza e objetividade dos indicadores. Na visão dos informantes-chave, os indicadores estão claros e objetivos. Quanto à necessidade de especificações numéricas, principalmente nos indicadores de processo, os informantes-chave consideraram que a forma apresentada (nominal, a exemplo de "realização do atendimento") estava adequada, pois a quantificação numérica inviabilizaria o consenso, uma vez que cada UPA apresentava metas específicas à luz de um contrato sendo estas repactuadas temporariamente. Contudo, realçamos como limitação deste estudo a pouca especificidade dos indicadores. Novos projetos deverão surgir para apri- 
moramento e validação desses elementos avaliativos mediante a necessidade local das UPAs.

Merecem a atenção os critérios descritos na subdimensão atuação complementar à atenção básica às urgências, pois refletem $\mathrm{o}$ atendimento fornecido durante $24 \mathrm{~h}$ pelas UPAs à população. Destacou-se que o indivíduo, muitas vezes, chega à UPA sem encaminhamento da atenção básica, tornando difícil o registro de tais casos. Na visão dos informanteschave, para a subdimenção SAMU 192, a integração é mais visível, todavia questionamentos sobre o registro dessa integração foram levantados. Diante da dificuldade dos registros que esta dimensão suscitou, conclui-se que possivelmente haverá problemas na fonte de verificação para mensurar tais indicadores. Contudo, entendeu-se que cada critério/ indicador possui um bom grau de importância, principalmente pelo fato de a UPA estar em uma posição intermediária entre as unidades básicas de saúde/ Saúde da Família e a rede hospitalar. 4

Neste estudo, os componentes foram interligados, visando à lógica do desenvolvimento da intervenção. Para seu delineamento, exigiu-se uma concentração no sentido de construir uma aproximação normativa a uma realidade prática. Desta

\section{Referências}

1. Santos JS, Scarpelinis S, Brasileiro SLL, Ferraz CA, Dallora MELV, Sá MFS. Avaliação do modelo de organização da Unidade de Emergência do HCFMRP-USP, adotando, como referência, as políticas nacionais de atenção às urgências e de humanização. Medicina (Ribeirão Preto). 2003; 36: 498-515.

2. Brasil. Ministério da Saúde. Portaria $n^{0} 2.048$ de 05 de novembro de 2002. Aprova o Regulamento Técnico dos Sistemas Estaduais de Urgências e Emergências. Diário Oficial da União; 2002.

3. Brasil. Ministério da Saúde. Portaria $n^{\circ} 2.922$ de 2 de dezembro de 2008. Propõe a implantação/adequação de Unidades de Pronto Atendimento - UPA. Diário Oficial da União; 2008.

4. Brasil. Ministério da Saúde. Portaria $n^{\circ} 1.020$ de 13 de maio de 2009. Estabelece diretrizes para a implantação do componente pré-hospitalar fixo (Unidades de Pronto Atendimento - UPA). Diário Oficial da União; 2009

5. Machado K. Novidade no SUS - Unidade de Pronto atendimento -UPA. Rev RADIS. 2009; 83: 12-6.

6. Thurston WE, Ramaliu A. Evaluability assessmentof a survivors of torture program: lessons learned. Can J Prog Eval. 2005; 20: 1-25.

7. Natal S, Samico I, Oliveira LGD, Assis AMJ. Estudo de avaliabilidade da rede de formação de recursos humanos da Secretaria de Vigilância em Saúde do Ministério da Saúde. Cad Saúde Colet. 2010. 18: 560-71.

8. Trevisan MS, Huang YM. Evaluability assessment: a primer. Prat Assess Res Eval. 2003; 8: 2-9. forma, buscou-se um modelo que explicita os consensos possíveis de um grupo de interessados em relação aos componentes fundamentais ao processo avaliativo. Nessa perspectiva, sua natureza não é definitiva, uma vez que sua construção é pautada no processo dinâmico que envolve a realidade da intervenção. Assim, concordando com Bezerra et al. $27 \mathrm{e}$ Medeiros et al., 25 o modelo lógico deve ser revisto e reconstruído periodicamente, podendo-se acrescentar ou retirar dados à medida que novas informações forem coletadas.

Os resultados do presente trabalho revelaram que as Unidades de Pronto Atendimento estudadas encontram-se adequadas à realização de avaliações, uma vez que se pôde verificar que os elementos identificados no modelo lógico são condizentes com as condições que a intervenção possui para alcançar suas metas e objetivos. Desta forma, o estudo traz como benefício maior a construção de subsídios para elaboração de ferramentas avaliativas com a participação ativa dos interessados, havendo troca de experiências entre as diferentes categorias profissionais.

9. Lima LRF, Silva LMV. Ampliação do acesso à atenção oftalmológica: um estudo sobre a avaliabilidade da campanha "De Olho na Visão". Ciênc Saúde Coletiva. 2008; 13 (Supl. 2): 2059-64.

10. Bezerra LCA, Alves CKA, Reis YAC, Samico I, Felisberto E, Carvalho ALB, Silva GS. Identificação e caracterização dos elementos constituintes de uma intervenção: pré-avaliação da política ParticipaSUS. Ciênc Saúde Coletiva. 2012; 17: 883-900.

11. Vanderheyden L, Verhoef M, Scott C, Pain K. Evaluability assessment as a tool for research network development: experiences of the complementary and alternative medicine education and research network of Alberta, Canada. Can J Prog Eval. 2006; 21: 63-82.

12. Thurston WE, Potvin L. Evaluabilitity asssessment: a tool for incorporating evaluation in social change programmes in evaluation. London, Thousand Oaks and New Delhi: SAGE Publications. 2003; 9: 453-69.

13. Brasil. Ministério da Saúde. Portaria no 1.863 de 29 de setembro de 2003. Institui a Política Nacional de Atenção às Urgências (PNAU). Brasília, DF; 2003.

14. Brasil. Ministério da Saúde. Política Nacional de Atenção às Urgências. Ministério da Saúde, Brasília, DF; 2004. 236p. (Série E. Legislação de Saúde).

15. Pernambuco. Secretaria de Saúde do Estado. Diretoria Geral de Assuntos Jurídicos. Contrato de Gestão n. 01/2010. Recife: Secretaria de Saúde do Estado de Pernambuco; 2010. 
16. Instituto de Medicina Integral Prof. Fernando Figueira. Relatório de avaliação semestral das metas contratualizadas da Unidade de Pronto Atendimento de Olinda - Janeiro a Junho/2010. Recife; 2010.

17. Reis YAC. Consensos sobre o papel do gestor estadual na regionalização da assistência à saúde do SUS [dissertação]. Recife: Centro de Pesquisas Aggeu Magalhães; 2010.

18. Contandriopoulos AP, Champagne F, Denis JL, Pineault RA. Avaliação na area da saúde: conceitos e métodos. In: Hartz ZMA (org). Avaliação em saúde: dos modelos conceituais à prática na análise da implantação de programas. Rio de Janeiro: Fundação Oswaldo Cruz (Fiocruz), 1997: 29-48.

19. Hartz ZMA, Contandriopoulos AP. Integralidade da atenção e integração de serviços de saúde: desafios para avaliar a implantação de um "sistema sem muros". Cad Saúde Pública. 2004; 20 (Supl. 2): S331-6.

20. Medina MG, Silva GAP, Aquino R, Hartz ZMA. Uso de modelos teóricos na Avaliação em Saúde: aspectos conceituais e operacionais. In: Hartz ZMA, Vieira da Silva LM. Avaliação em saúde: dos modelos teóricos à prática na avaliação de programas e sistemas de saúde. Salvador/ Rio de Janeiro: EDUFBA/FIOCRUZ; 2005. p. 41-64.

21. Souza LEPF, Silva LMV, Hartz ZMA. Conferência de consenso sobre a imagem-objetivo da descentralização da atenção à saúde no Brasil. In: Hartz ZMA, Vieira da Silva LM, orgs. Avaliação em saúde: dos modelos teóricos à prática na avaliação de Programas e Sistemas de Saúde. Salvador/ Rio de Janeiro: EDUFBA/FIOCRUZ; 2005. P. 41-74.

22. Worthen RB, Sanders JR, Fitzpatrick JL. Identificação e seleção de perguntas e critérios. In: Avaliação de programas: concepções e práticas. São Paulo: Editora Gente, 2004; p. 341-71.

23. Stufflebeam DL. O modelo CIPP para avaliação. In: Instituto Fonte, org. Introdução à avaliação de programas sociais. São Paulo: Instituto Fonte; 2004. (Coletânea de textos).

24. Thurston WE, Graham J, Hatfieleld J. Evaluability assessment: a catalyst for program change and improvement. Eval Health Prof. 2003; 26: 206-21.

25. Medeiros PFP, Bezerra LCA, Santos NTV, Melo EO. Um estudo sobre avaliabilidade do Programa + Vida: política de redução de danos em álcool, fumo e outras drogas do município de recife, Brasil. Rev Bras Saúde Matern Infant. 2010; 10 (Supl. 1): S 209-17.

26. Mclaughlin JA, Jordan GB. Logic models: a tool for telling your program's performance story. Eval Prog Plan. 1999; 22: 65-72.

27. Bezerra LCA, Cazarin G, Alves CKA. Modelagem de programas: da teoria à operacionalização. In: Samico I, Felisberto E, Figueiró AC, Frias PG, orgs. Avaliação em saúde: bases conceituais e operacionais. Recife: Medbook; 2010. p. $65-78$

28. Vuori H. A Qualidade em saúde. Avaliação da atenção básica: construindo novas ferramentas para o SUS. Divulg Saúde Debate. 2000; 21: 15-28.

29. Leviton LC, Khan LK, Rog D, Dawkins N. Cotton D. Evaluability assessment to improve public health policies, programs, and practices. Ann Rev Public Health. 2010; 31 : 213-33.

30. Andrés CP. ¿Deben estar las técnicas de consenso incluídas entre las técnicas de investidación cualitativa? Rev Esp Salud Pública. 2000; 74: 319-21.

31. Kaplan SA, Garrett KE. The use of logic models by community-based initiatives. Eval Prog Plan. 2005; 28: $167-72$

32. Renger R, Titcomb A. A three-step approach to teaching logic models. Am J Eval. 2002; 23: 493-503.

Recebido em 3 de setembro de 2012

Versão final apresentada em 5 de outubro de 2012

Aprovado em 31 de outubro de 2012 